\title{
Investigation of proteomic signatures related to gender, age and body mass index
}

\author{
A. Curran ${ }^{1}$, M. Kutmon ${ }^{2}$, C.T. Evelo ${ }^{2}$, S.L. Coort ${ }^{2}$, M. F. Ryan ${ }^{1}$, E.R. Gibney ${ }^{1}$, M.J. Gibney ${ }^{1}$, \\ H.M. Roche ${ }^{1}$ and L. Brennan ${ }^{1}$ \\ ${ }^{1}$ Institute of Food and Health, School of Agriculture and Food Science, University College Dublin, Dublin 4, Ireland \\ and ${ }^{2}$ Department of Bioinformatics - BiGCaT, Maastricht University, Maastricht, The Netherlands
}

Proteomics has played an important role in advancing nutrition research in recent years ${ }^{(1)}$. The area has strong potential to contribute towards the identification of new biomarkers in nutrition research, however understanding the impact of phenotypic and biochemical variables on the proteomic signature is key to doing this. The primary objectives of this research are to 1) examine the impact of phenotypic parameters on the proteomic signature and 2) uncover pathways enriched by the significant proteins.

This research focuses on data obtained from the Metabolic Challenge (MECHE) study. Healthy participants aged 18-60 years were recruited, and baseline samples from those who completed an OGTT and had complete proteomic data, were selected $(\mathrm{n}=100)$. The MECHE dataset contains information on 1129 proteins measured using the SOMAscan assay from SomaLogic. The impact of gender, age and body mass index (BMI) on the proteomic signature was assessed using principal component analysis (PCA) and partial least squares discriminant analysis (PLS-DA). Pathway analysis was performed using PathVisio ${ }^{(2)}$. This study was performed according to the Declaration of Helsinki.

PLS-DA models demonstrated that gender, age and BMI all had an effect on the proteomic profile of the 100 healthy individuals. Based on the PLS-DA models, males and females were discriminated with an $\mathrm{R}^{2}$ of 0.870 , and a $\mathrm{Q}^{2}$ of 0.550 . Age categories were discriminated with an $\mathrm{R}^{2}$ of 0.741 and a $\mathrm{Q}^{2}$ of 0.159 , while $\mathrm{BMI}$ categories were discriminated with an $\mathrm{R}^{2}$ of 0.614 and a $\mathrm{Q}^{2}$ of $0 \cdot 314$. When variable importance of projection values $\geq 2$ were taken from the PLS-DA models, 40 proteins were associated with gender, 36 with ageing and 28 with BMI. Associated molecular functions and biological processes were extracted from the UniProt database and representative pie charts were created. Pathway analysis revealed altered pathways for gender, age and BMI. Bone metabolism pathways such as osteoblast signalling were significantly altered by age related proteins, along with complement pathways and micronutrient pathways (see table below).

\begin{tabular}{|c|c|c|c|c|}
\hline Pathway (WikiPathway Identifier) & \# Proteins/total & Z Score & $P$-value & $\begin{array}{c}\% \text { of proteins measured } \\
\text { by assay }\end{array}$ \\
\hline Complement Activation (WP545) & $8 / 17$ & $5 \cdot 57$ & $0 \cdot 001$ & $77 \cdot 3$ \\
\hline Complement and Coagulation Cascades (WP558) & $12 / 38$ & $5 \cdot 00$ & $0 \cdot 001$ & $64 \cdot 4$ \\
\hline Osteoblast Signalling (WP322) & $3 / 8$ & $2 \cdot 85$ & $0 \cdot 031$ & $57 \cdot 1$ \\
\hline Vitamin B12 Metabolism (WP1533) & $6 / 25$ & $2 \cdot 68$ & $0 \cdot 011$ & $49 \cdot 0$ \\
\hline Overview of nanoparticle effects (WP3287) & $3 / 9$ & $2 \cdot 58$ & $0 \cdot 026$ & $47 \cdot 4$ \\
\hline Allograft Rejection (WP2328) & $7 / 36$ & $2 \cdot 26$ & $0 \cdot 027$ & $46 \cdot 2$ \\
\hline Folate Metabolism (WP176) & $6 / 27$ & $2 \cdot 46$ & $0 \cdot 019$ & $40 \cdot 9$ \\
\hline Selenium Micronutrient Network (WP15) & $6 / 28$ & $2 \cdot 36$ & $0 \cdot 020$ & $33 \cdot 7$ \\
\hline Osteoclast Signalling (WP12) & $2 / 6$ & $2 \cdot 10$ & 0.035 & $37 \cdot 5$ \\
\hline
\end{tabular}

Pathway statistics in PathVisio revealed significantly altered pathways by age related proteins $(\mathrm{Z}$-score $>1.96, P$-value $<0 \cdot 05)$. The number of proteins (\#proteins/total) represent the number of differentially expressed proteins in the pathway compared to the total number of measured proteins by SOMAscan assay in the pathway.

To our knowledge, this is the first study to give an overview of proteins related to each of the phenotypic parameters gender, age and BMI in a healthy cohort. Future work will investigate the overlap of significant proteins for these phenotypic parameters and the potential links between proteins and health status.

1. de Roos B, McArdle HJ (2008) Br J Nutr. 99(3): S66-71.

2. Kutmon M, van Iersel MP, Bohler A et al. (2015) PLoS Comput Biol. 11(2): e1004085. 